\title{
INVERSE TECHNIQUE FOR VORTEX IMAGING AND ITS APPLICATION TO FEEDBACK FLOW CONTROL
}

\author{
Takao Suzuki, Tim Colonius, and Douglas G. MacMartin ${ }^{\dagger}$ \\ Division of Engineering and Applied Science \\ California Institute of Technology, Pasadena, California 91125
}

\begin{abstract}
A simple vortex imaging algorithm using a least square method is proposed for feedback flow control. The position and the circulation of a vortex convected in a channel are identified from the time history of pressure at a limited number of points on the wall. The capabilities of the algorithm are demonstrated using two-dimensional direct numerical simulations. A few observer points on one side of the wall are sufficient to detect the position and the circulation of a compact vortex to a reasonable degree of accuracy. This inverse algorithm is introduced to feedback separation control for a diffuser flow. The algorithm is modified for a curved channel, and the so-called "estimator-corrector" and "fast algorithm" are applied to reduce the uncertainty of prediction with less computational time. The DNS results demonstrate that the feedback control using the inverse technique can enhance the robustness against unsteady disturbances and lessen stagnation pressure loss.
\end{abstract}

\section{INTRODUCTION}

Inverse problems have been extensively investigated in many fields, such as solid mechanics, acoustics, electro-magnetics, etc. However, only a very few inverse problems have been investigated in fluid mechanics (Derou, et al. 1995; Gumerov \& Chahine 2000; Bonnet, et al. 1998; and others) because of the nonlinearity of the governing equations. Although some structure identification techniques based on surface pressure have been investigated (Chang, Piomelli, \& Blake 1999; Naguib, Wark, \& Juckenhöfel 2001; Murray \& Ukeiley 2002), these methodologies have focused on statistical approaches.

For real time feedback flow control, for example, we wish to rapidly extract the key structure of in-

Copyright (c)2003 by Takao Suzuki, Tim Colonius, and Douglas G. MacMartin. Published by the American Institute of Aeronautics and Astronautics, Inc., with permission. stantaneous flow fields from a limited number of sensors. However, the current flow reconstruction techniques, such as proper orthogonal decomposition (Lumley 1970) or stochastic estimation (Adrian $\&$ Moin 1988), require the information from nearly the entire flow field. Suzuki \& Colonius (2003) proposed a reconstruction algorithm using a least square method for a vortex convected in a channel. From a limited number of observer points on one side of the wall, the position and the circulation of a vortex can be estimated. Hence, the database of the flow field is not needed a priori. Moreover, to suppress the uncertainty of prediction, vortex dynamics is taken into account (called the "estimator-corrector"), and the algorithm is simplified to rapidly detect a vortex (called the "fast algorithm"). This type of non-invasive technique is potentially applicable to detect distinctive flow structures, such as streamwise vortices from trailing edges or on delta wings, separated bubbles in a diffuser, rolling-up vortices in a cavity; thus, it is relevant to active feedback flow control.

In this paper, we review the inverse method developed by Suzuki \& Colonius (2003) and introduce it into feedback separation control using unsteady mass injection in a diffuser. Local mass injection can suppress stagnation pressure loss and flow distortion by pinching off the separated boundary layer into vortices with an appropriate size. Although periodic injection (i.e. open-loop) has been shown to be more effective than steady forcing with fixed authority, closed-loop control is necessary to maintain performance in the presence of unpredictable disturbances. Previous feedback control techniques have only varied forcing parameters at a rate much slower than the frequency of large-scale flow unsteadiness (e.g. Kwong \& Dowling 1994). We establish closedloop control on the time-scale of vortex shedding in a diffuser and examine it using direct numerical simulation (DNS) in two-dimensions. We monitor a vortex growing near the separation point using the inverse method; subsequently, we issue a pulse in- 
AIAA Paper 2003-4260

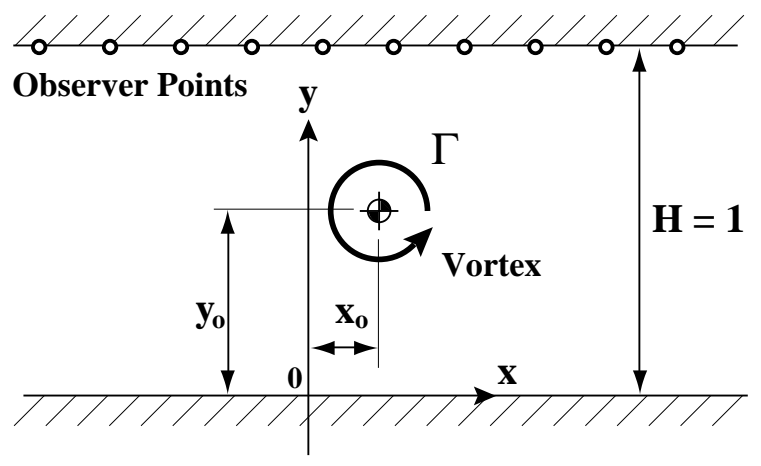

Figure 1: Schematic of the model problem.

jection when its circulation exceeds a critical value. Thus, we can enhance the robustness of unsteady separation control.

The outline of the paper is as follows: In the next section, the inverse vortex imaging algorithm is reviewed, and the architecture of feedback separation control is described. Next, the procedures of the numerical simulations are presented. Subsequently, the algorithms are validated based on DNS, and the closed-loop control is examined.

\section{INVERSE ALGORITHMS}

In this paper, we review algorithms only needed for the present feedback flow control problem. Refer to Suzuki \& Colonius (2003) for more detailed study of the inverse algorithms.

\section{IMAGING A SINGLE VORTEX}

We consider a single point vortex convected in a channel and assume an ideal fluid (see figure 1 for the coordinate system). A limited number of observer points are distributed only on the upper wall $(y=1)$. The position and circulation of the vortex is to be detected only from the pressure time history at these points. The position in the horizontal direction can be easily identified from pressure deficit on the wall induced by the vortex. The difficulty is to determine the circulation and the height of the vortex.

For an ideal fluid the analytic solution to the forward problem can be found (c.f. Saffman 1992). We define the physical domain as $z=x+i y$ and use a conformal mapping expressed by $\zeta=\exp \left(\frac{\pi}{2} z\right)$ : We can map an infinitely long channel into the first quadrant, and two pairs of counter-rotating vortices satisfy the non-penetration boundary conditions in the $\zeta$ domain. After some calculation the convective velocity of the vortex in the physical domain can be given as a function of $y_{0}$ :

$$
u_{c}=\frac{\Gamma}{4} \frac{1}{\tan \left(\pi y_{o}\right)} .
$$

If the vortex approaches the upper wall, the induced velocity of the image vortex on the upper side becomes stronger so that the real vortex moves to the left, and vice versa. Thus, if the convective velocity can be estimated from the time dependent pressure data, it can in turn be used to estimate the height of the vortex.

The pressure fluctuation on the wall due to the vortex can be readily calculated using Bernoulli's equation:

$$
\begin{aligned}
p\left(\Gamma, x_{o}, y_{o} ; x\right) & =p_{\infty}-\frac{\rho \Gamma^{2}}{2}\left[\left(\frac{\eta^{2} \sin 2 \theta}{\left(\eta^{2}+1\right)^{2}-4 \eta^{2} \sin ^{2} \theta}\right)^{2}\right. \\
& \left.+\frac{1}{2 \tan 2 \theta} \frac{\eta^{2} \sin 2 \theta}{\left(\eta^{2}+1\right)^{2}-4 \eta^{2} \sin ^{2} \theta}\right],(2)
\end{aligned}
$$

where $p_{\infty}$ is the ambient pressure in the far field (assumed known), $\theta \equiv \frac{\pi}{2} y_{o}$, and $\eta \equiv \exp \left[\frac{\pi}{2}\left(x-x_{o}\right)\right]$. Subsequently, the derivative of the pressure with respect to time can be expressed as

$$
\dot{p}\left(\Gamma, x_{o}, y_{o} ; x\right)=\frac{\partial p}{\partial x_{o}} \frac{d x_{o}}{d t}=-\frac{\partial p}{\partial x}\left(u_{\infty}+u_{c}\right),
$$

where $u_{\infty}$ is the freestream velocity in the channel and $u_{c}$ is given by (1). The strategy is to find the position and the circulation which best match (2) and (3) simultaneously.

To evaluate this, we introduce a cost function of the least square type:

$$
\begin{aligned}
& J\left(t, \Gamma, x_{o}, y_{o}\right)= \\
& \sum_{m=1}^{N_{m}}\left[w_{1}\left(\Gamma, x_{o}, y_{o} ; q\right)\left|p\left(\Gamma, x_{o}, y_{o} ; x_{m}\right)-q\left(t, x_{m}\right)\right|^{2}\right. \\
& \left.+w_{2}\left(\Gamma, x_{o}, y_{o} ; \dot{q}\right)\left|\dot{p}\left(\Gamma, x_{o}, y_{o} ; x_{m}\right)-\dot{q}\left(t, x_{m}\right)\right|^{2}\right],
\end{aligned}
$$

where $N_{m}$ denotes the number of the observer points, $x_{m}$ and $q\left(t, x_{m}\right)$ are the position and measured pressure for the $m^{\text {th }}$ observer, respectively. The first term in (4) mainly determines the horizontal coordinate, $x_{o}$, and the second term the vertical coordinate, $y_{o}$. Here, we simply set the weight functions $w_{1}$ and $w_{2}$ so that the relative errors from both terms are equal and they depend only on $q$ and $\dot{q}$, respectively:

$$
w_{1}(q)=\left(\sum_{m=1}^{N_{m}}\left|q\left(t, x_{m}\right)-p_{\infty}\right|^{2}\right)^{-1}
$$




$$
w_{2}(\dot{q})=\left(\sum_{m=1}^{N_{m}}\left|\dot{q}\left(t, x_{m}\right)\right|^{2}\right)^{-1} .
$$

To minimize $J$, we differentiate (4) with respect to $\Gamma$. This must satisfy

$$
\frac{\partial J}{\partial \Gamma}=2 \sum_{m=1}^{N_{m}}\left[w_{1} \frac{\partial p}{\partial \Gamma}(p-q)+w_{2} \frac{\partial \dot{p}}{\partial \Gamma}(\dot{p}-\dot{q})\right]_{m}=0,
$$

where the subscript $m$ indicates that the quantity is evaluated at the $m^{\text {th }}$ observer position. Equation (7) gives the estimated circulation, $\Gamma^{*}$, if the vortex is located at $(x, y)=\left(x_{o}, y_{o}\right)$. Substituting $\Gamma^{*}$ into (4), we can compute the cost function at each point and map it on the $\left(x_{o}, y_{o}\right)$ domain. Thus, we create a "vortex image map" on which the local minimum of $J\left(t, \Gamma^{*}, x_{o}, y_{o}\right)$ indicates the vortex position.

\section{IMAGING A VORTEX BASED ON APPROXIMATED SOLUTIONS}

When a vortex is convected in a curved flow, the potential solution may not be expressed by a simple formula. In such a case we can approximate the potential solution by a family of simple functions with several parameters. As an example, we consider a curved channel in which the coordinate in the flow direction is given by $\xi$ and that in the transverse direction by $\eta$ (see figure 2). From the previous discussion, we observe that the position of the pressure minimum approximately gives the $\xi$ coordinate and the width of this pressure deficit strongly depends on the $\eta$ coordinate. In addition, as the circulation increases, the pressure deficit enhances. From such observation, suppose we model the exact solution on the upper wall using the following formula:

$\tilde{p}\left(a, b, c ; \Gamma, \xi_{o}, \eta_{o} ; \xi_{m}\right) \equiv \bar{p}\left(\xi_{m}\right)-\frac{a \Gamma^{c}}{b\left(1-\eta_{o}\right)} \mathrm{e}^{-\frac{\left(\xi_{m}-\xi_{o}\right)^{2}}{b^{2}\left(1-\eta_{o}\right)^{2}}}$,

where $\bar{p}\left(\xi_{m}\right)$ denotes the pressure for the $m^{\text {th }}$ observer when a vortex is absent.

Now, if the potential solutions with several different combinations of $\left(\Gamma, \xi_{o}, \eta_{o}\right)$ are available from computation (the total number of the samples is denoted by $N_{n}$ ), we can determine the coefficients, $a$, $b$, and $c$, by minimizing the following cost function:

$$
\begin{aligned}
& \tilde{J}(a, b, c) \equiv \\
& \sum_{n=1}^{N_{n}} \varpi_{n} \sum_{m=1}^{N_{m}} \mid \tilde{p}\left(a, b, c ; \Gamma_{n},\left(\xi_{o}\right)_{n},\left(\eta_{o}\right)_{n} ; \xi_{m}, \eta_{m}\right) \\
& -\left.p\left(\Gamma_{n},\left(\xi_{o}\right)_{n},\left(\eta_{o}\right)_{n} ; \xi_{m}, \eta_{m}\right)\right|^{2},
\end{aligned}
$$

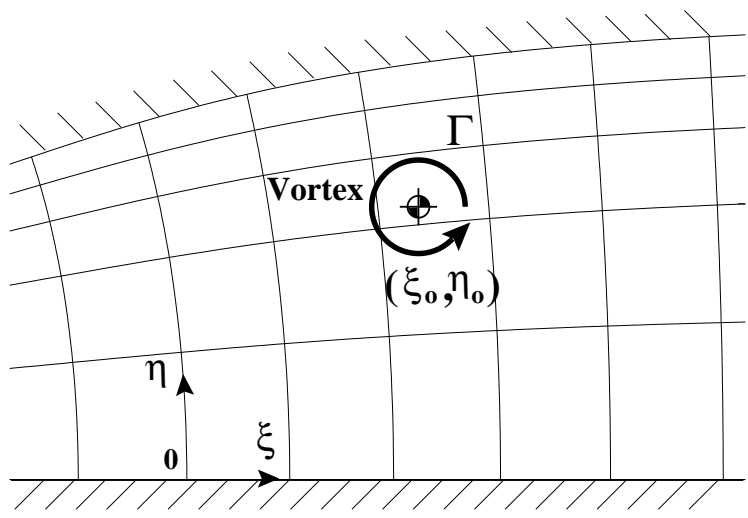

Figure 2: Coordinate system in a curved channel.

where $p$ is again the potential solution, and $\varpi_{n} \equiv$ $\left(\sum_{m=1}^{N_{m}}\left|p\left(\Gamma_{n},\left(\xi_{o}\right)_{n},\left(\eta_{o}\right)_{n} ; \xi_{m}, \eta_{m}\right)-\bar{p}\left(\xi_{m}\right)\right|^{2}\right)^{-1}$ for convenience.

Similarly, the time derivative $\tilde{\dot{p}}$ could be approximated by some model function. In this study, however, assuming that the mapping function from the curvilinear coordinates $(\xi, \eta)$ to the physical coordinates $(x, y)$ is known, we use the same convective velocity derived in (1) and the relation (3) in the $(\xi, \eta)$ domain. In addition, we assume that the freestream velocity (denoted by $u_{\infty}$ in (3)) is aligned with an iso $\eta$ line. Determining $a, b$, and $c$, we can use $\tilde{p}$ and $\tilde{\dot{p}}$ instead of $p$ and $\dot{p}$ and follow the same procedure to create vortex image maps.

\section{ESTIMATOR-CORRECTOR}

If we keep track of the vortex position and circulation over time, we can take the flow dynamics into account by using the information from the previous time step; accordingly, we can suppress the random errors associated with the detection process. Defining the state vector to be $\mathbf{x} \equiv\left(\Gamma, x_{o}, y_{o}\right)^{T}$, e to be the error of $\mathbf{x}, \mathbf{u} \equiv \dot{\mathbf{x}}$ given from the potential solution, and $\delta \mathbf{u}$ to be the error of $\mathbf{u}$, we can express the governing equation at the time step $i+1$ to be

$$
\mathbf{x}_{i+1}=\mathbf{x}_{i}+\mathbf{u}_{i} \Delta t
$$

where $\mathbf{u}_{i} \equiv \mathbf{u}\left(\mathbf{x}_{i}\right)$ and the error associated with temporal discretization is neglected. The corresponding equation estimated via the observers can be expressed as

$$
\begin{aligned}
\mathbf{x}_{i+1}+\mathbf{e}_{i+1} & =r\left[\mathbf{x}_{i}+\mathbf{e}_{i}+\left(\mathbf{u}_{i}+\delta \mathbf{u}_{i}\right) \Delta t\right] \\
& +(1-r)\left[\mathbf{x}_{i+1}+\mathbf{w}_{i+1}\right]
\end{aligned}
$$

where $0<r<1$ and $\mathbf{w}_{i+1}$ denotes the random error vector associated with the inverse process at the time 
AIAA Paper 2003-4260

step $i+1$. Here, the first term gives the prediction based on the vortex dynamics and the second term gives the detection from the inverse algorithm. From (10) and (11) the recursive relation of the error term yields

$$
\mathbf{e}_{i+1} \approx r\left(I+\Delta t \frac{\partial \mathbf{u}}{\partial \mathbf{x}}\right) \mathbf{e}_{i}+(1-r) \mathbf{w}_{i+1} .
$$

If $\mathbf{w}_{i}$ is independent and identical with the mean zero, the expectation of the error at the time step $n$ yields

$$
E\left[\mathbf{e}_{n}^{2}\right]=\left[\hat{r}^{2 n}+(r-1)^{2} \frac{\hat{r}^{2 n}-1}{\hat{r}^{2}-1}\right] E\left[\mathbf{w}^{2}\right],
$$

where $E\left[\mathbf{e}_{n}^{2}\right] \equiv\left((\delta \Gamma)_{n}^{2},\left(\delta x_{o}\right)_{n}^{2},\left(\delta y_{o}\right)_{n}^{2}\right)^{T}$ and $\hat{r} \equiv r$. $\max \left[\right.$ eigenvalues of $\left.\left(I+\Delta t \frac{\partial \mathbf{u}}{\partial \mathbf{x}}\right)\right]$. Thus, as $n \rightarrow \infty$, we should choose $r$ so that $\hat{r}<1$ but close to unity. The deviation can be then suppressed as $E\left[\mathbf{e}_{\infty}^{2}\right] \approx$ $(1-r)^{2} /\left(1-\hat{r}^{2}\right) E\left[\mathbf{w}^{2}\right]$.

\section{FAST ALGORITHM}

When the ideal solution is a linear function of a parameter with respect to which we optimize the cost function, we can directly solve (7) without iteration. Suppose we use (8) as the ideal solution and define the cost function only in terms of $\tilde{p}$ (i.e. without $\tilde{\dot{p}}$ and thereby $w_{1}$ and $w_{2}$ ) for simplicity. Accordingly, we can optimize the cost function as

$$
\frac{\partial J}{\partial \Gamma^{c}}=2 \sum_{m=1}^{N_{m}}\left[\frac{\partial \tilde{p}}{\partial \Gamma^{c}}(\tilde{p}-q)\right]_{m}=0 .
$$

Thus, the optimum circulation can be obtained as

$$
\Gamma=\left[\frac{\sum_{m} \frac{a}{b\left(1-\eta_{o}\right)} \mathrm{e}^{-\frac{\left(\xi_{m}-\xi_{o}\right)^{2}}{b^{2}\left(1-\eta_{o}\right)^{2}}}(\bar{p}-q)_{m}}{\sum_{m} \frac{a^{2}}{b^{2}\left(1-\eta_{o}\right)^{2}} \mathrm{e}^{-2 \frac{\left(\xi_{m}-\xi_{o}\right)^{2}}{b^{2}\left(1-\eta_{o}\right)^{2}}}}\right]^{1 / c}
$$

This technique can substantially reduce computational time; hence, it is useful for real-time feedback flow control.

\section{FEEDBACK ARCHITECTURE}

Now, we introduce the inverse algorithm into feedback flow control. In this study, we consider separation control in a diffuser using unsteady mass injection. When the flow includes no external disturbances, periodic mass injection (i.e. open-loop forcing) with sufficient authority induces frequency locking and lessens stagnation pressure loss as well as

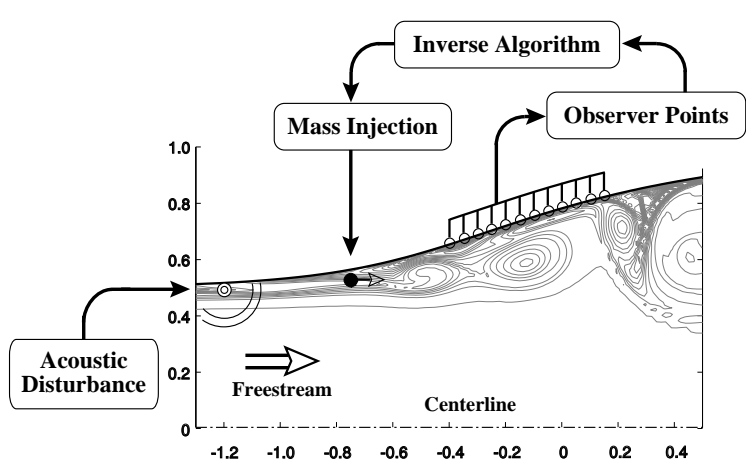

Figure 3: Schematic of the feedback loop. Vorticity contour near the separation point is displayed.

flow distortion. Previous studies (e.g. Suzuki, Colonius, \& Pirozzoli 2003) have indicated that a key to optimize the periodic separation control for internal flows is the size (circulation) of vortices when they are pinched off. However, unpredictable unsteady disturbances may break the uniformity of the vortex size and deteriorate the diffuser performance.

To enhance the robustness of the unsteady separation control, we introduce the inverse technique and monitor the motion of a vortex growing near the separation point. We use an approximated solution for the curved wall geometry near the separation point and the estimator-corrector as well as the fast algorithm to reduce uncertainty of prediction with less computational cost. Subsequently, we issue a pulse injection near the separation point and pinchoff vortices when their circulation exceeds a critical value (see figure 3 for a schematic of the feedback architecture). We determine the critical value based on a parametric study of open-loop periodic forcing with no disturbances. Here, we impose an acoustic point source far upstream of the separation point for unsteady disturbances.

\section{NUMERICAL PROCEDURES}

\section{DIRECT NUMERICAL SIMULATION (DNS)}

To demonstrate the capabilities of the inverse algorithms, we performed direct numerical simulations (DNS) of two-dimensional diffuser flows. First, we validated the algorithms using simple geometries and subsequently examined feedback control. The conditions common to both cases are first described in this section.

The diffuser shape is given by the following conformal mapping:

$$
z \equiv x+i y=\frac{3}{4}(\zeta+0.4400 \log [\cosh (0.7576 \zeta)])
$$




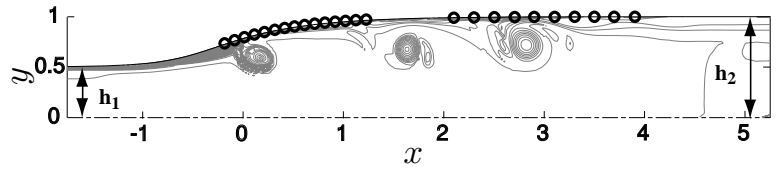

Figure 4: Whole computational domain with vorticity contours. The observer points used for validation are denoted by $\circ$. The group on the left hand side (15 points) is on the curved part, and the right hand side (10 points) is on the straight part.

where $\zeta \equiv \xi+i \eta$ (see figure 4 for the actual geometry). This yields the area ratio to be $h_{1} / h_{2}=0.5$ (where the subscripts 1 and 2 denote the inlet and exit, respectively).

To non-dimensionalize the equations, the exit height, $h_{2}$, is taken to be the length scale and the freestream velocity at the exit, $u_{2}$, to be the velocity scale. The exit Reynolds number was set to be $R e=4000$, and the momentum thickness of the inlet laminar boundary layer to be $\delta_{b . l}=0.025$.

We artificially forced the boundary layer near the separation point, $(x, y)=(-0.75,0.52)$, to control the size and phase of vortices. We imposed the nonslip boundary conditions on the upper wall and the symmetric conditions on the lower side. Further details regarding the numerical procedures can be found in Suzuki, Colonius, \& Pirozzoli (2003).

\section{INVERSE ALGORITHM FOR VALIDATION}

For validation purposes, we performed two test cases. For both cases, we distributed observer points on the upper wall with approximately equal spacing in the $x$ direction. We used the observer points downstream for a straight channel problem, while those in the upstream part for a curved channel problem (c.f. figure 4).

The exit freestream Mach number was set to be nearly incompressible $\left(M_{2} \approx 0.15\right)$. The pressure data were sampled at the computation time step of $\Delta t=1.5 \times 10^{-4}$. The ambient pressure $p_{\infty}\left(=p_{2}\right)$ and the freestream convective velocity $u_{\infty}\left(=u_{2}\right)$ were measured at $x=4$. before vortices were convected, and assumed to be given in the reconstruction process. We iteratively solved (7) using the Newton method. The resolution of the target domain was set to be $\Delta x=\Delta y=0.01(\Delta \xi=\Delta \eta=$ 0.01 for the curved channel problem).

For a curved channel problem, we used a family of functions expressed in (8) for the ideal solution and assumed that the mapping function of the diffuser shape (16) is known. To determine the coefficients $a, b$, and $c$, nine sample points (depicted in figure 5) times three values of circulations $(\Gamma=0.333,0.667$,

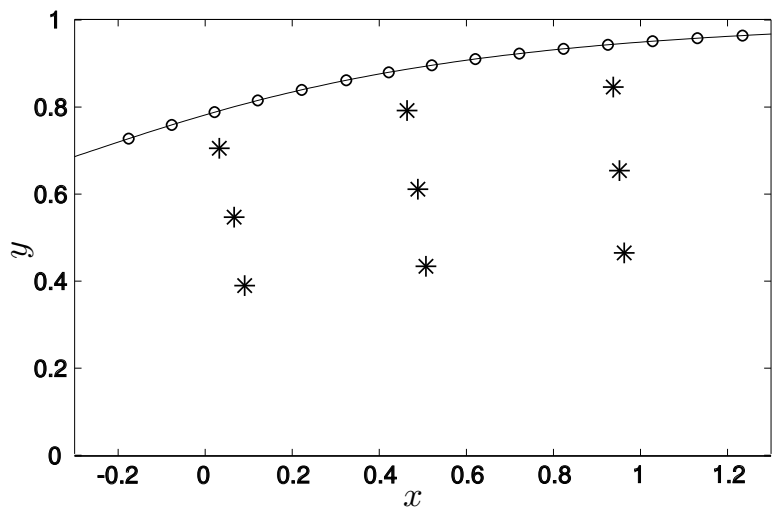

Figure 5: Points where the samples of the potential solutions were taken (denoted by $*$ ) for the validation case.

and 1.333), i.e. a total of 27 samples of the potential solutions for a point vortex, were solved, and the pressure profiles at the observer points were computed based on the unsteady Bernoulli's equation. These potential solutions were used in minimizing the cost function shown in (9), which gave $a=1.98 \times 10^{-4}, b=0.678$, and $c=1.626$.

In addition, the estimator-corrector was tested in the straight channel problem. It was assumed that the circulation of each vortex is conserved and it is convected horizontally with the velocity of $u_{2}+u_{c}$ $\left(u_{c}\right.$ is given by (1)). The weight was set to be $r=0.9$ in (11) so that $\hat{r}<1$ is satisfied.

\section{FEEDBACK LOOP}

To examine the feedback loop, the simulation was performed in a shorter domain $(-1.75 \leq x \leq 2.75$ in figure 4) and the inflow Mach number was set to be $M_{1}=0.4\left(M_{2}=0.2\right)$. For an inflow disturbance, a time harmonic point source at $\omega_{\text {acoust }} h_{2} / u_{2}=10.5$ was imposed at $(x, y)=(-1.200,0.485)$ (c.f. figure 3). This forcing frequency was found to significantly deteriorate performance of the open-loop forcing. The amplitude of pressure generated by this source was approximately $\left|\Delta p / p_{1}\right| \sim 2 \times 10^{-4}$ near the separation point. Refer to Suzuki \& Lele (2003) for the detailed procedures of the acoustic forcing $\left(A_{p} \sim 1.5 \times 10^{-3}\right.$ in their (3.2)-(3.4)).

Twelve observer points were distributed slightly downstream of the separation point, and similar procedures were taken to determine the approximate solution for the curved geometry (c.f. figure 20 shown later). Here, the fast algorithm was used with setting $a=5.48 \times 10^{-3}, b=0.600$, and $c=2$ in (15). The estimator-corrector was activated only when circulation is monotonically increasing, and it was assumed that vortices are convected along iso 


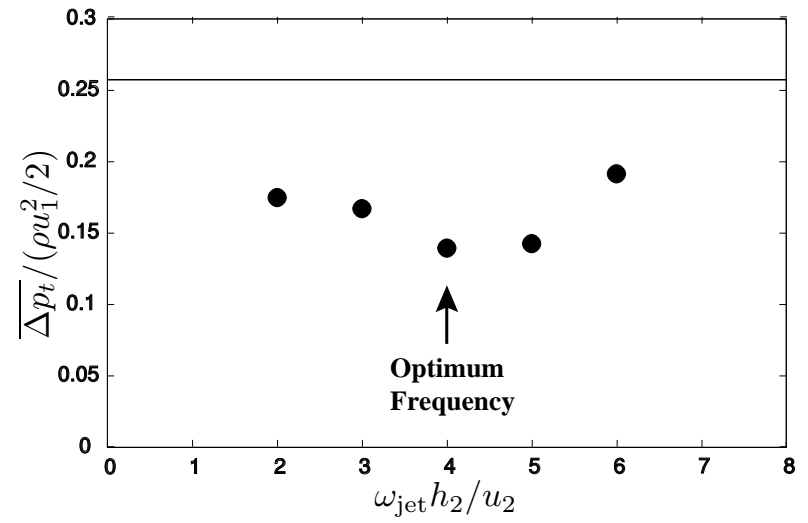

Figure 6: Stagnation pressure loss in nondisturbance operations. The horizontal line denotes the unforced case. The data were taken after the section averaged stagnation pressure loss became periodic (c.f. figure 21).

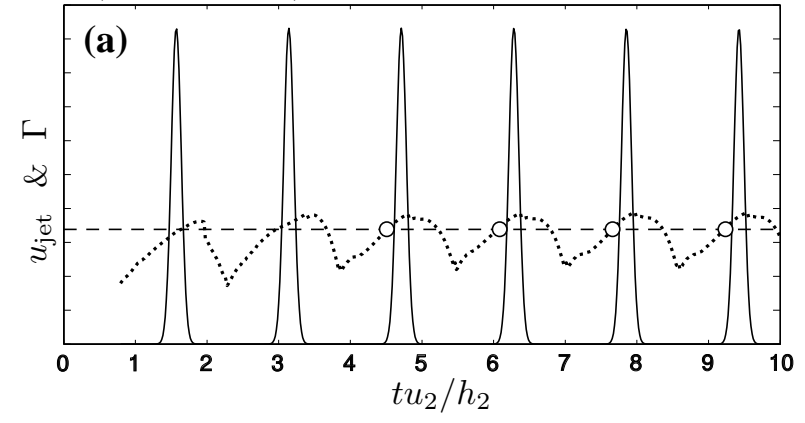

Figure 7: Phase relation between the injection velocity (denoted by —) and the estimated circulation (denoted by ....) of separation vortices at the optimum frequency $\left(\omega_{\text {jet }} h_{2} / u_{2}=4\right.$. $) . \quad u_{\text {jet }}$ and $\Gamma$ are arbitrary scaled. The critical value of circulation is denoted by 0 .

$\eta$ lines and the rate of circulation increase is given by $\frac{d \Gamma}{d t}=\frac{u_{1}^{2}-u_{2}^{2}}{2}$ (an incompressible limit, c.f. Suzuki, Colonius, \& Pirozzoli 2003) during this period. The pressure data were sampled at the computation time step of $\Delta t=3.2 \times 10^{-2}$, and the ambient pressure and the freestream convective velocity were given from the potential solution.

Next, we determine the critical value of circulation at which a pulse injection starts. Periodic Gaussian pulse injections were simulated at several different frequencies with fixed authority $\left(C_{\rho} \equiv\right.$ $\bar{u}_{\text {jet }} h_{s} /\left(u_{1} h_{1}\right)=0.16 \%$ where $h_{s}$ is the slot width $)$. Refer to figure 6 for the comparison of stagnation pressure loss. Referring to the phase relation between the injection and the circulation in the optimum case $\left(\omega_{\text {jet }} h_{2} / u_{2}=4\right.$. $)$, we determined the critical circulation to be $\Gamma=1.69$ (c.f. figure 7 ).

\section{RESULTS AND DISCUSSION}

\begin{tabular}{|c|c|c|}
\hline \hline Case & $\begin{array}{c}\text { Straight channel } \\
(5,10,20 \text { pts. })\end{array}$ & Curved channel \\
\hline$\sqrt{\overline{(\Delta x)^{2}}}$ & $0.048,0.015,0.013$ & 0.014 \\
$\sqrt{\overline{(\Delta y)^{2}}}$ & $0.104,0.044,0.037$ & 0.057 \\
$\sqrt{\overline{(\Delta \Gamma / \Gamma)^{2}}}$ & $0.369,0.191,0.183$ & 0.326 \\
\hline \hline
\end{tabular}

Table 1: Standard deviation of position and circulation. Three values in the second column were calculated using 5, 10, and 20 points, respectively.

\section{DETECTION OF A SINGLE VORTEX IN A STRAIGHT CHANNEL}

First, we show the results of compact vortices detected using the regular algorithm with 5, 10, and 20 observer points. Figures $8 \sim 11$ depict image maps of one and two vortices and their comparisons between the detected vortex positions using 10 points and the vortex centroids computed from DNS superposed on the vorticity contours. They demonstrate that the position of the vortex is identified very well (within 0.03 in both examples). Even two vortices can be captured in a single map if they are sufficiently separated. Here, the vortex centroid and the circulation in DNS were calculated by defining the vortex region to be within a simple support up to $3 \%$ of the peak vorticity.

We can confirm the accuracy of detection from the trajectories of the vortex and the circulation in figures 12 and 13, respectively. We also tabulate the standard deviations of the position and the circulation in table 1. Although the position tends to be predicted quite well (within 0.05 using 10 points), the accuracy of the circulation is relatively low (as low as 20\%). Such a trend can be understood from the following analysis: For example, if a point vortex of $\Gamma=1.0$ is located at $\left(x_{o}, y_{o}\right)=(3.0,0.5)$, it gives $\left(\frac{\partial^{2} J}{\partial \Gamma^{2}}, \frac{\partial^{2} J}{\partial x_{0}^{2}}, \frac{\partial^{2} J}{\partial y_{o}^{2}}\right)=(16.0,67.9,23.7)$ at the optimum point; namely, the resolution of $\Gamma$ is several times as low as those of $x_{o}$ and $y_{o}$. This also explains that the contour maps of $-J$ tend to be elongated in the $y$ direction.

Figures 12 and 13 indicate that the deviations of detection are less sensitive to the number of the observer points if it is sufficient (say, more than 10 points in this example); namely, the error barely vanishes. They also show that the prediction deteriorates as the vortex exists near the inlet or the exit.

Figures 12 and 13 imply that as the position is mis-predicted upward, the circulation is underesti- 


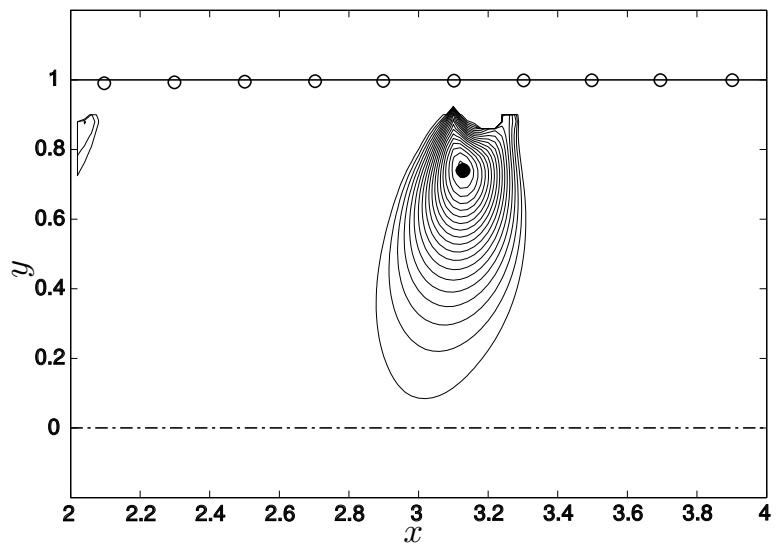

Figure 8: Image map of a vortex created using the regular vortex algorithm. Contours of $-J$ corresponding to figure 9 are drawn with the interval of $\Delta J=0.05$. The detected position is denoted by $\bullet$, and the observer points are denoted by $\circ$. The cost function at the detected point is $J_{\min }=0.886$.

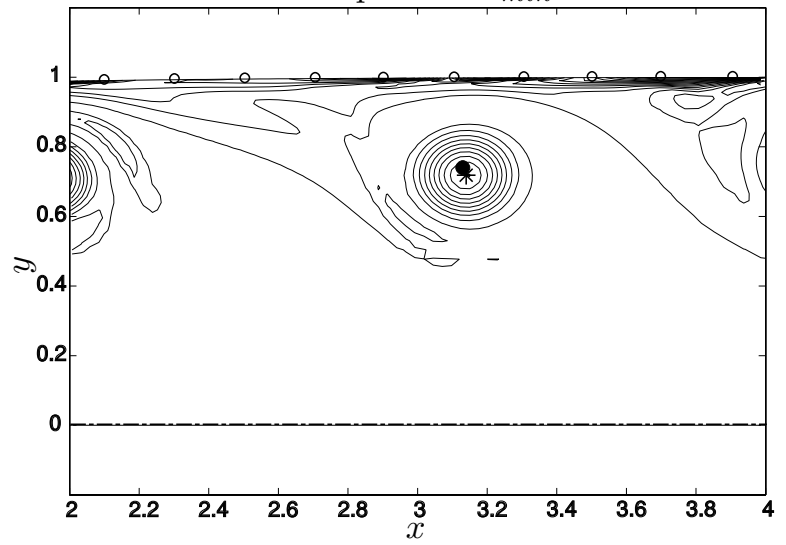

Figure 9: Detected vortex position using the regular vortex algorithm with vorticity contours. The estimated circulation is $\Gamma=0.759$ as opposed to $\Gamma=0.909$ from DNS. The vortex centroid is denoted by $*$. The rest of the notation is the same as figure 8 .

mated, and vice versa. This tendency can be readily derived from the optimization process.

\section{DETECTION IN A CURVED CHANNEL}

Figures 14 and 15 depict the results from the curved channel case. Although the ideal solution is only very approximate in this case, the algorithm captures the main vortex to nearly the same degree of accuracy (within 0.06 in position) as the straight channel case. The small vortex growing near the separation point also appears on the map although the detected position is not accurate. Figure 16 depicts the comparison of the vortex trajectories and shows that the vertical coordinate tends to be estimated slightly higher (by 0.057 from table 1 ). It also indicates that the algorithm becomes unstable

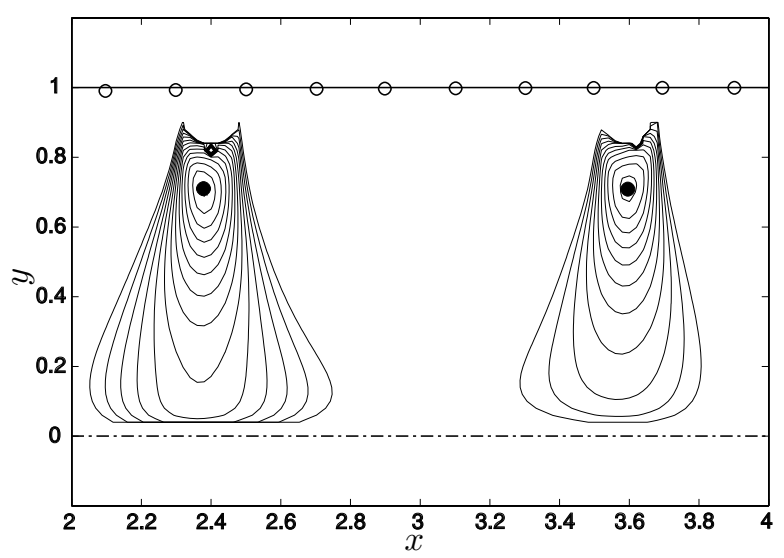

Figure 10: Image map of two vortices created using the regular vortex algorithm. The corresponding vorticity contour is shown in figure $11 . J_{\min }=1.007$ and 1.133 on the left and right hand sides, respectively. Notation is the same as figure 8 .

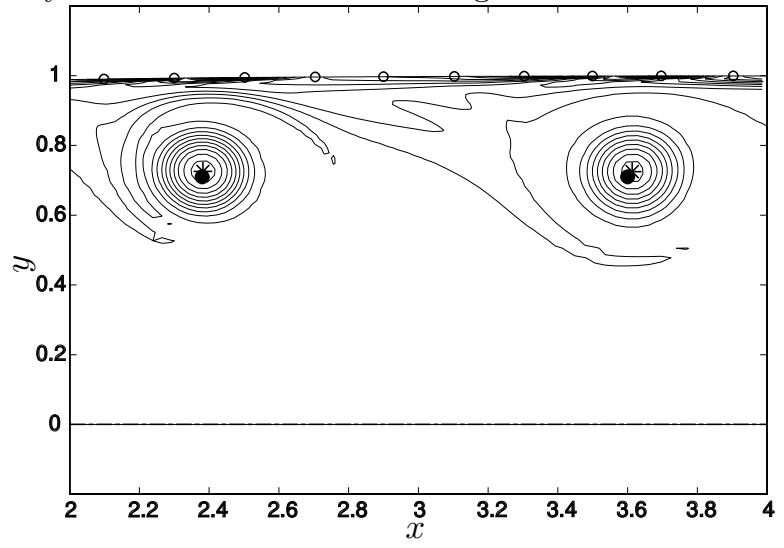

Figure 11: Detected positions of two vortices using the regular vortex algorithm with vorticity contours. The estimated circulations are $\Gamma=0.997$ and $\Gamma=$ 0.995 as opposed to $\Gamma=0.946$ and $\bar{\Gamma}=0.889$ for the left and right vortices, respectively. Notation is the same as figure 9 .

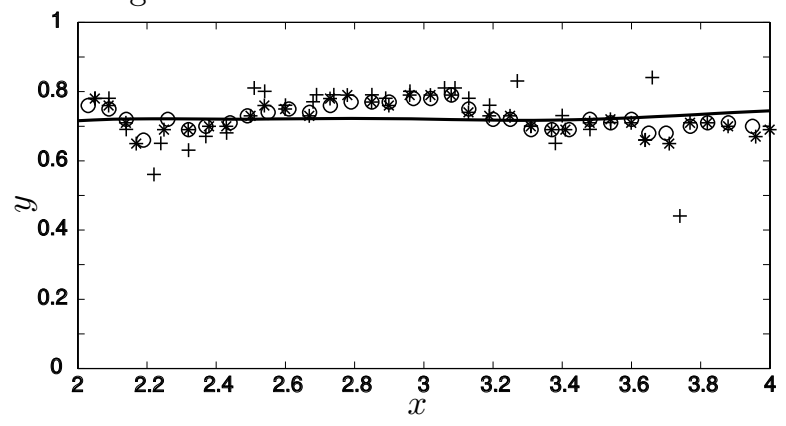

Figure 12: Trajectory of a vortex in a straight channel: - , vortex centroid; +++ , detected positions from 5 observer points; $* * *$ those from 10 points; and $\circ \circ \circ$, those from 20 points. 


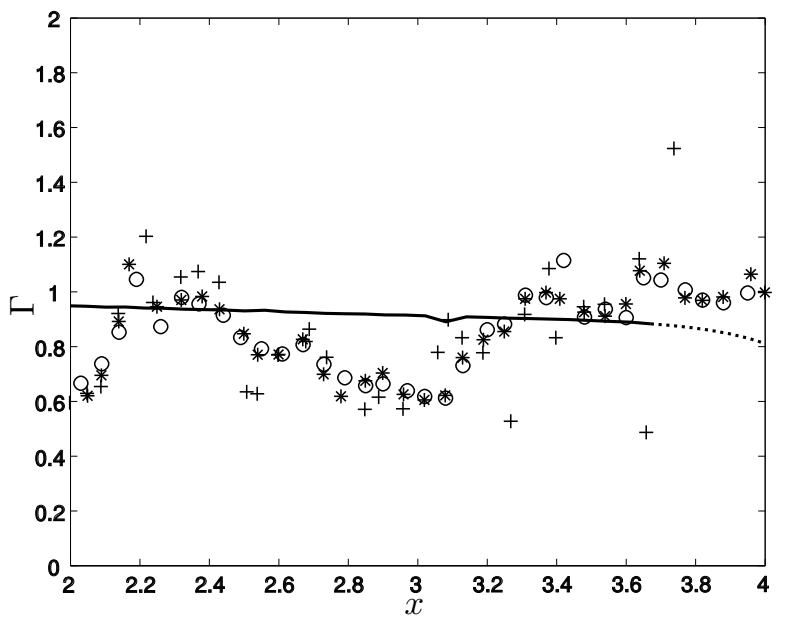

Figure 13: Circulation change of a vortex in a straight channel. Notation is the same as figure 12 . Dotted portion indicates that the leading edge of the vortex enters the computational sponge at the exit.

when the vortex is too close to the separation point. Pressure on the wall near the separation point fluctuates largely depending on whether the boundary layer is attached or not. The magnitude of this fluctuation tends to exceed that of the pressure deficit induced by the vortex. Figure 17 shows that the circulation is underestimated by $33 \%$ on average (from table 1) although the decaying trend is qualitatively correct. Since the approximate solution is assumed proportional to a power of $\Gamma$ as expressed in (8), the unsteady part of pressure cannot be appropriately represented by this expression.

\section{TRAJECTORIES PROCESSED BY ESTIMATOR-CORRECTOR}

Figure 18 compares the raw vortex trajectory with that processed using the estimator-corrector for the straight channel case (the same case as figure 12). Since the estimator-corrector includes the vortex dynamics, the trajectory becomes much smoother and the deviation tends to decrease in time. Figure 19 compares the circulation change and shows similar trends although it starts with large deviation.

\section{FEEDBACK SEPARATION CONTROL}

We first study the accuracy of the fast inverse algorithm. Figure 20 shows the comparison of the vortex trajectories: one obtained from DNS and the others from the prediction with and without the estimator-corrector. The accuracy substantially decreases as the vortex grows and moves downstream, mainly because its distribution is elongated in the flow direction. Since the vortex is still attached in the boundary layer, its circulation is not well-defined

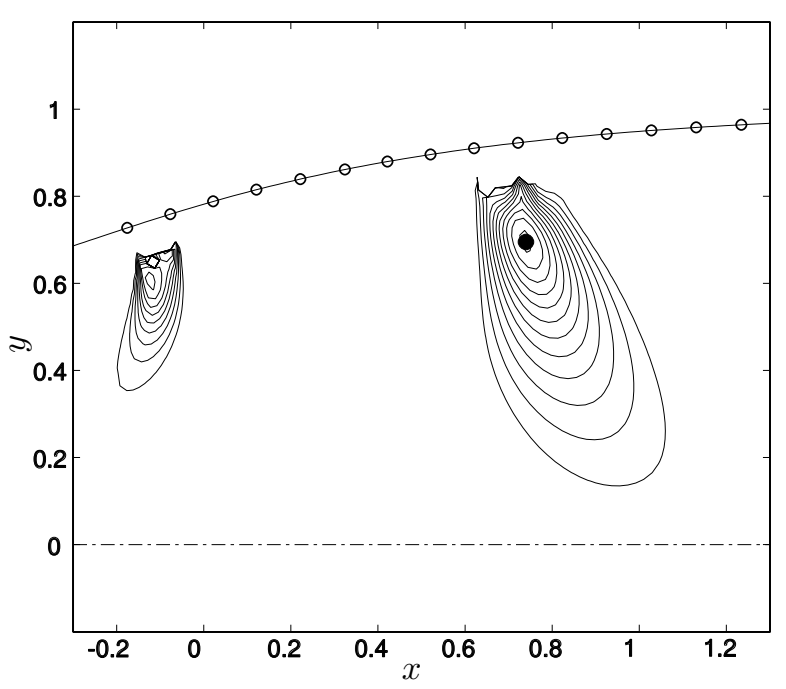

Figure 14: Image map created based on an approximated solution in a curved channel (with 15 points). $J_{\min }=1.218$. Notation is the same as figure 8 .

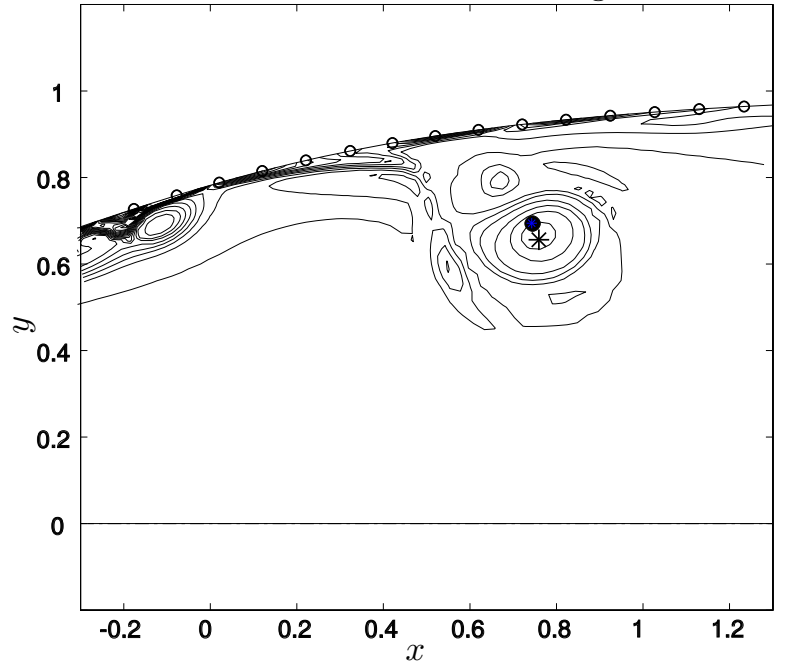

Figure 15: Vorticity contour with the detected vortex position in a curved channel. The estimated circulation is $\Gamma=0.684$, while that computed from DNS is $\Gamma=1.035$. Notation is the same as figure 9 .

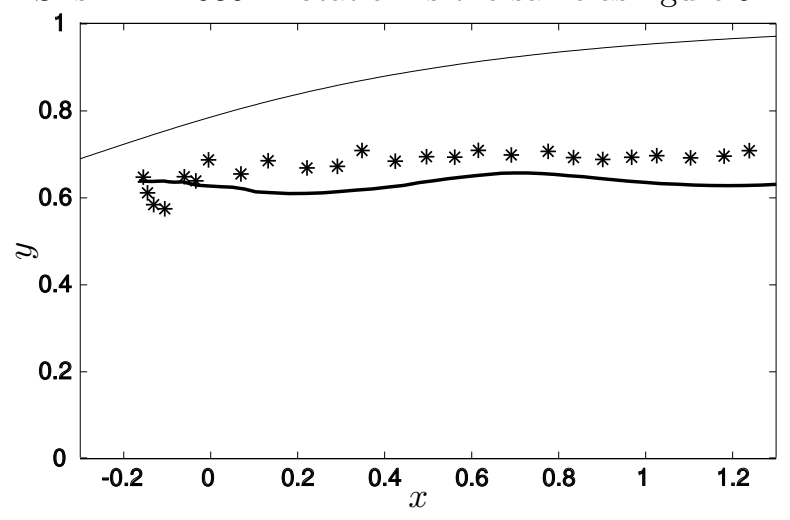

Figure 16: Trajectory of a vortex in a curved channel. Notation is the same as figure 12 (detected from 15 points). 


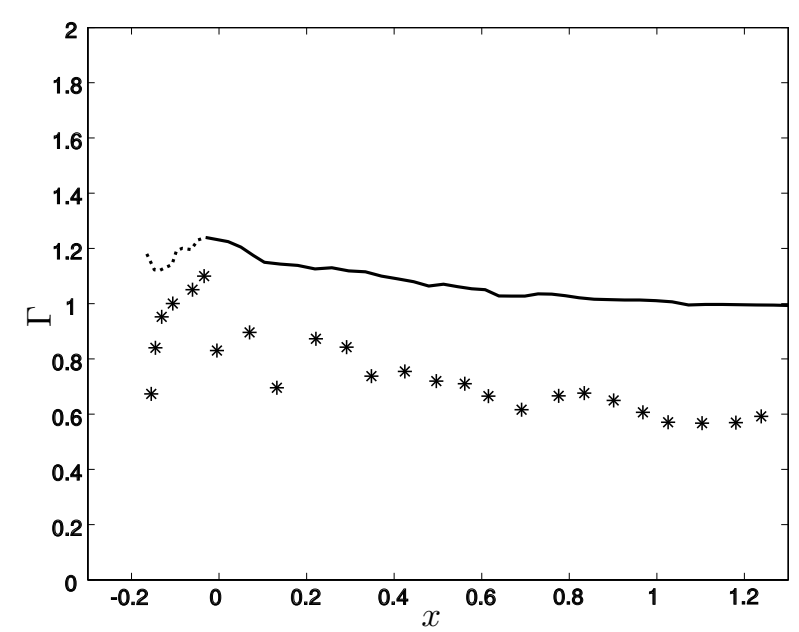

Figure 17: Circulation change in a curved channel. Notation is the same as figure 13. Dotted line indicates that the circulation cannot be well defined because the vortex is still attached to the boundary layer.

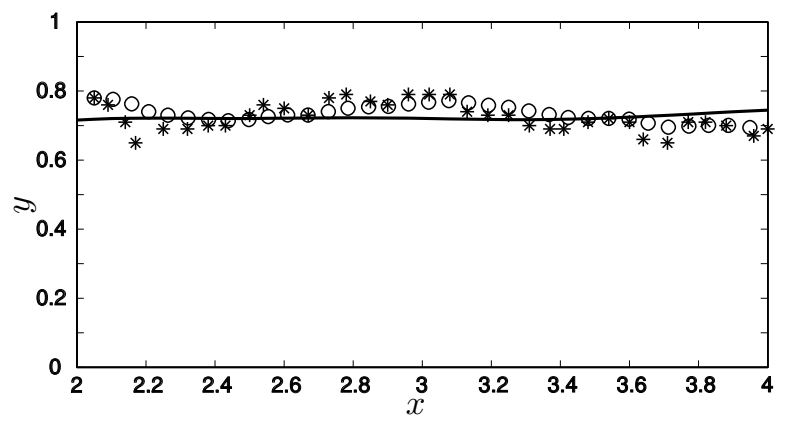

Figure 18: Comparison of trajectories in a straight channel: —, vortex centroid; $* * *$, regular algorithm; ০০০, with the estimator-corrector. Ten points are used.

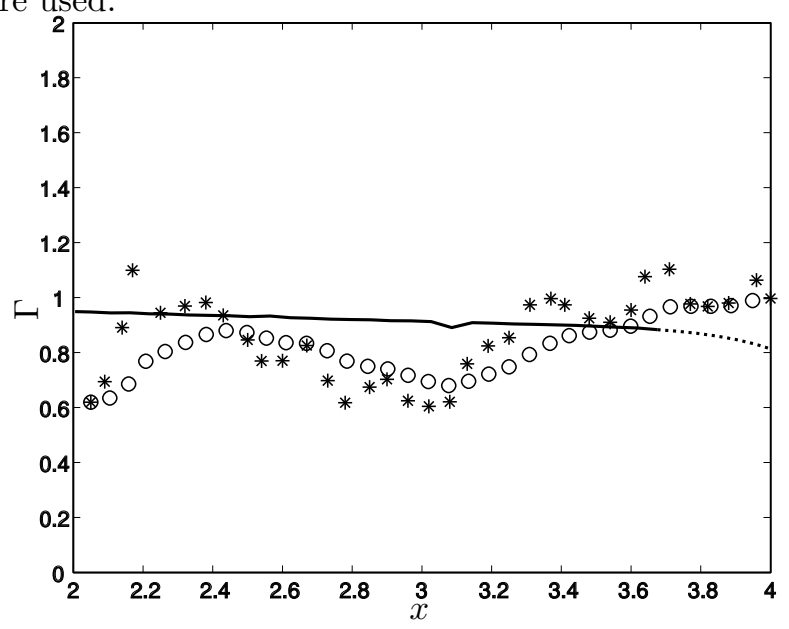

Figure 19: Comparison of circulation change in a straight channel. Notation is the same as figure 18 .

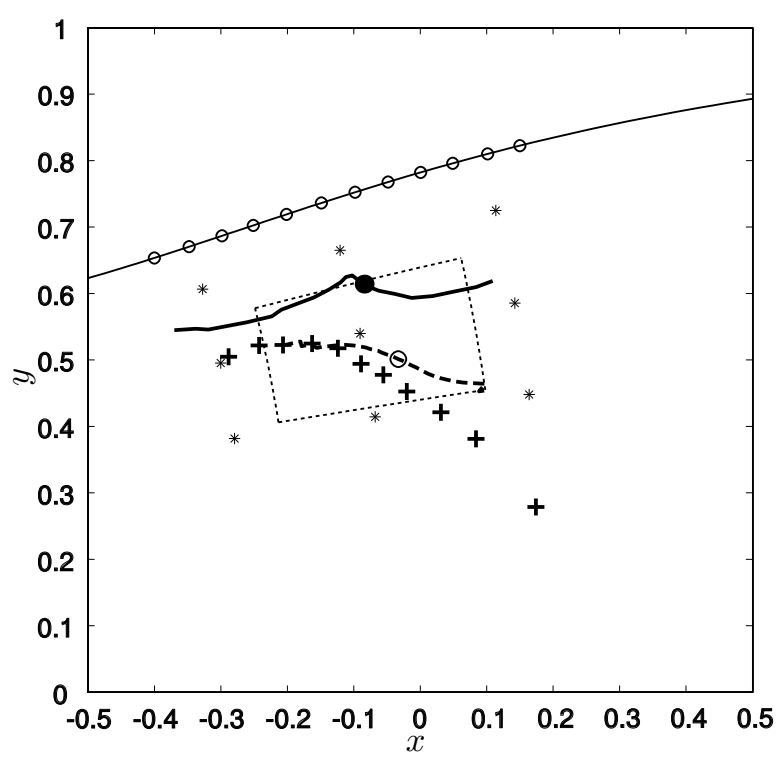

Figure 20: Vortex trajectories near the separation point (open-loop forcing at $\omega_{\text {jet }} h_{2} / u_{2}=4$.): local vorticity peaks given from DNS; +++ , the prediction using the fast inverse algorithm; $--_{-}$, that with the estimator-corrector. The position at which the injection starts is indicated by $\bullet$ on the vorticity peak trajectory and by $\circ$ on the estimated one. The monitored domain is denoted by.... . The rest of the notation is the same as figure 5 .

and was not measured here. Note that the trajectory given from DNS indicates a kink due to vortex pairing.

Next, we observe the robustness against an inflow acoustic disturbance. Figure 21 shows stagnation pressure profiles of the non-disturbance case at the optimum injection frequency, the same injection frequency with a disturbance, and the same disturbance with feedback control, respectively. The inflow acoustic disturbance irregulaizes frequency locking, which is invoked by an open-loop forcing in the non-disturbance case; as a result, stagnation pressure recovery significantly deteriorates $\left(\overline{\Delta p_{t}} /\left(\rho u_{1}^{2} / 2\right)=0.146 \rightarrow 0.205\right.$ from the nondisturbance to the disturbed case during the period indicated in figure 21). However, the feedback loop can re-induce frequency locking and recovers stagnation pressure loss in the same degree as the nondisturbance case (to $\left.\overline{\Delta p_{t}} /\left(\rho u_{1}^{2} / 2\right)=0.149\right)$. The feedback loop rapidly adjusts a harmonic of the disturbance frequency $\left(3 \omega_{\text {acoust }}\right)$ in this case. Thus, although the estimate based on the fast inverse algorithm is not accurate, we expect that the actual circulation of the pinched-off vortices in case (c) is approximately equal to that in case (a).

Figure 22 shows the phase relation between the 

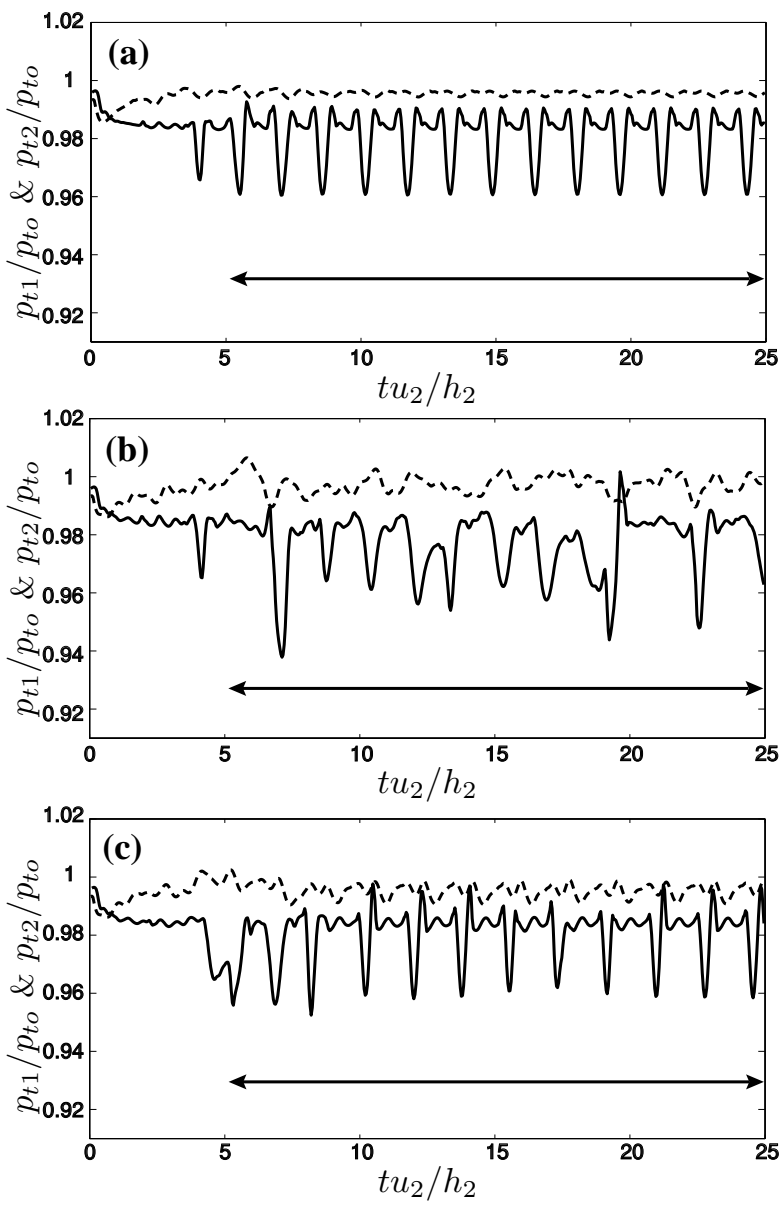

Figure 21: Comparison of stagnation pressure profiles. Stagnation pressure averaged over the inlet cross section $(x=-1)$ is denoted by --- , and that over the exit $(x=1.5)$ is - : (a) non-disturbance case at the injection frequency of $\omega_{\text {jet }} h_{2} / u_{2}=4$., (b) the same injection frequency with the inlet acoustic disturbance, and (c) the same inlet acoustic disturbance with feedback control, from top to bottom.

injection and the estimated circulation. In the openloop case, injections do not necessarily pinch-off vortices (declination of circulation indicates that a vortex is pinched-off and convected out of the domain). In the feedback case, a pulse is held until the circulation grows beyond the critical value so that the flow is regularized.

\section{CONCLUSIONS}

This paper has reviewed the capabilities of the inverse algorithms using a least square cost function based on two-dimensional DNS. Although the accuracy of prediction in circulation is not yet high $(\sim 30 \%)$, the position is detected reasonably well $\left(\sim 0.05 h_{2}\right)$. We may be able to apply such a general
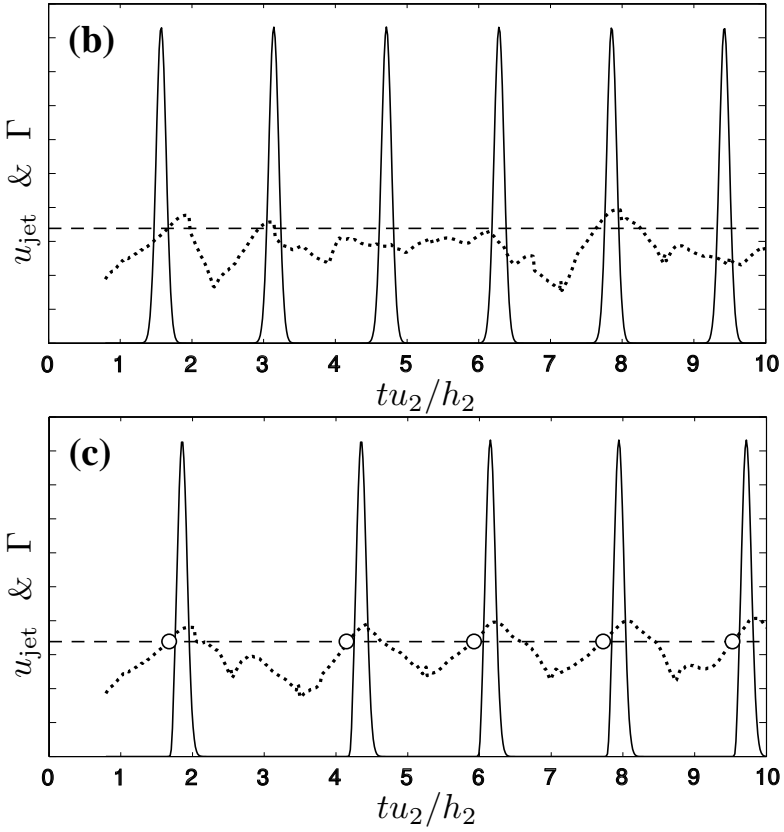

Figure 22: Phase relations between the injection velocity and the estimated circulation of separation vortices for the disturbed cases: (b) open-loop injection; (c) feedback injection. (Non-disturbance case, (a), was shown in figure 7 .)

inverse algorithm not only for vortical flow, but also in many other applications. When the key structures of the flow field are somewhat known, this inverse algorithm can extract an instantaneous flow field only from the information on the boundary.

Not surprisingly, the accuracy of the detection depends on the suitability of the ideal solution. One way to overcome this problem is to introduce more parameters, such as a core radius of a vortex, and to optimize the cost function in terms of this parameter. Another way is to construct a better representation referring to specific flow conditions. For example, we can modify the ideal solution by considering the eccentricity of a separation vortex.

The suitability of the ideal solution becomes crucial as we solve for more realistic flow fields. For example, if we apply this algorithm in threedimensions using the solution for a line vortex, the resultant image map may capture large-scale line vortices with small curvatures. However, when the flow is more complex, we may need to include additional parameters for curvature (or to consider vortex rings instead) and to use a multiple vortex algorithm (c.f. Suzuki \& Colonius 2003 for the multivortex algorithm). As the numbers of the objects and the parameters to be optimized increase, the convergence of optimization becomes less stable and 
takes more time. Thus, the algorithm requires further improvement for practical applications.

The inverse algorithm has been applied to feedback separation control on the time scale of flow unsteadiness. The accuracy of estimate using the fast inverse algorithm needs to be improved. Since this simplified algorithm eliminates the timedependence, the estimate in the transverse direction becomes poor in particular. Nonetheless, the DNS results indicate the closed-loop control can enhance the robustness against unsteady disturbances.

Unlike conventional feedback control (i.e. techniques analyzed in the frequency domain or in a state-space), such a feedback control technique only requires a simple phase relation in terms of one parameter (i.e. circulation). On the other hand, the operation is not fully optimized although the optimum frequency is searched to determine the critical circulation. For example, including the information on the vortex position, the temporal wave-form of injection could be optimized. To investigate these aspects, further study on separation dynamics is required.

\section{$\underline{\text { ACKNOWLEDGMENTS }}$}

We appreciate Prof. Richard Murray for an idea of the estimator-corrector and Mr. Kazuo Sone for generating several files. The work on inverse algorithms described here was supported by DARPA (Defense Advanced Research Projects Agency) program (contract number F49620-00-C-0035).

\section{REFERENCES}

Adrian, R. J. \& Moin, P. 1988 Stochastic estimation of organized turbulent structure: homogeneous shear flow. J. Fluid Mech. 190, 531-559.

Bonnet, J. P., et al. 1998 Collaborative testing of eddy structure identification methods in free turbulent shear flows. Experiments in Fluids, 25, 197225 .

Chang, P. A., Piomelli, U., \& Blake, W. K. 1999 Relationship between wall pressure and velocity-field sources. Phys. Fluids, 11, no.11, 3434-3448.

Derou, D., Dinten, J-M., Herault, L., \& Niez, J-J. 1995 Physical-model based reconstruction of the global instantaneous velocity field from velocity measurement at a few points. Proceedings of the workshop on physics-based modeling in computer vision, 63-69.
Gumerov, N. A. \& Chahine, G. L. 2000 An inverse method for the acoustic detection, localization and determination of the shape evolution of a bubble. Inverse Problems, 16, 1741-1760.

Kwong A. H. M. \& Dowling, A. P. 1994 Active boundary-layer control in diffusers. AIAA J. 32, no.12, 2409-2414.

Lumley, J. L. 1970 Stochastic Tools in Turbulence, Academic Press.

Murray, N. E. \& Ukeiley, L. S. 2002 Estimating the shear layer velocity field above an open cavity from surface pressure measurements. AIAA paper, AIAA2002-2866.

Naguib, A. M., Wark, C. E., \& Juckenhöfel, O. 2001 Stochastic estimation and flow sources associated with surface pressure events in a turbulent boundary layer. Phys. Fluids, 13, no.9, 2611-2626.

Saffman, P. G. 1992 Vortex Dynamics, Cambridge University Press.

Suzuki, T. \& Colonius, T. 2003 An inverse imaging method for detection of a vortex in a channel. accepted for $A I A A J$.

Suzuki, T., Colonius, T., \& Pirozzoli, S. 2003 Twodimensional diffuser model for vortex shedding and DNS study on periodic separation control. submitted to J. Fluid Mech.

Suzuki, T. \& Lele, S. K. 2003 Green's functions for a source in a boundary layer: direct waves, channelled waves and diffracted waves. J. Fluid Mech. 477, $129-173$. 